\title{
Some Inferential Aspects of Finite Population Sampling With Additional Resources
}

\author{
Samindranath Sengupta \\ Calcutta University, Calcutta, India \\ Bikas K. Sinha \\ Indian Statistical Institute (Calcutta), India \\ and \\ North Carolina State University, Raleigh, U.S.A. \\ and \\ Sastry G. Pantula \\ North Carolina State University, Raleigh, U.S.A.
}

\begin{abstract}
The probicm of extending a given sampling design, when additional resources are available, is considered. Some existing methods of improving an initial sampling strategy, so that the use of the additional resources is justified, are criticaliy revieved. Admissibility of the existing strategies is questioned. In the process, inproved strategies are suggested in various cases.
\end{abstract}

AMS 1980 Subject classification: Primary 62005 Secondary 62C10

Key words and phrases: Finite Population Sampling Designs, Extension Rules, Adnissible Strategies. 


\section{INTRODUCTION}

Suppose a survey statistician is interested in estimating a parametric function $\theta(Y)$ of a character $Y$ for a finite labelled population $U_{\sim}=(1,2, \ldots, N)$ of size N. Given the initial resources, the statistician has decided to adopt the strategy $\left(P_{n}, e_{n}\right)$ where $P_{n}$ refers to a fixed sample design of size $n$ and $e_{n}$ refers to an estimator for $\theta(\underline{Y})$. Let $s_{n}$ denote a typical sample of size $n$ from $U$ and $S_{n}$ denote the sample space consisting of samples of the type $S_{n}$ for which $P_{n}\left(s_{n}\right)$ is positive. Additional resources are subsequently made available to the statistician which may be used to obtain another sample of $k$ units. We assume throughout $n+k<N$. In this paper we discuss the choices for the sampling design for the second sample. We also present reasonable sampling strategies $\left(P_{n+k}, e_{n+k}\right)$ based on the combined sample.

We use the following notations and definitions in this paper.

(i) An estimator $e_{n}$ is said to be $\left(P_{n}-\right)$ unbiased for $\theta(Y)$ if

$$
s_{n} \sum_{\varepsilon} s_{n} e_{n}\left(s_{n}\right) P_{n}\left(s_{n}\right)=\theta(Y)
$$

for all $Y=\left(Y_{1}, \ldots, Y_{N}\right)$.

(ii) A strategy ( $P$, e) is said to be unbiased for $\theta(Y)$ if the estimator e is $(P-)$ unbiased for $\theta(Y)$ in the above sense.

(iii) An unbiased sampling strategy $(P, e)$ is said to be at least as good as another unbiased sampling strategy $\left(P^{*}, e^{*}\right)$ if

$$
\sum_{s \in S} e^{2}(s) P(s) \leq \sum_{s \in S^{*}} e^{\star^{2}}(s) P *(s)
$$

for all $Y$, where $S$ and $S^{*}$ are the sample spaces corresponding to the sampling designs $P$ and $P^{*}$ respectively. We say $(P, e)$ is better than $\left(P^{*}, e^{*}\right)$ if strict inequality holds in (1.1) for at least one $Y$. The sampling strategy $(P, e)$ is said to be admissible if there is no other sampling strategy that is better than 
$(P, e)$. It is said to be inadmissible otherwise. In this paper we consider unbiased strategies only.

Let $P_{k}$ denote a fixed size sampling design of size $k$ on $U_{\sim}$. Suppose the statistician uses the sampling design $P_{n}$ to obtain $s_{n}$ and when additional resources are available, uses $P_{k}$ to obtain an independent sample $s_{k}$. Then the combined sample, $s_{n} U s_{k}$, may be of size varying from $n$ to $n+k$. We denote the sampling design obtained by taking an independent sample in the second stage by $P_{n} U P_{k}$. Given $s_{n}$ is selected in the first stage using the sampling design $P_{n}$, an alternative procedure is to select a sample of size $k$ from $\underline{\sim}_{-} s_{n}$. Let $\left\{Q_{k}\left(\cdot \mid s_{n}\right) \mid s_{n} \varepsilon S_{n}\right\}$ denote a family of such fixed size sampling designs of size $k$. If the second sample $s_{k}$ is selected from $I-s_{n}$ using $Q_{k}\left(\cdot \mid s_{n}\right)$, following the selection of $s_{n}$ as the first stage sample, then the combined sample $s_{m}$ is of fixed size $m=n+k$. Let $P_{m}$ denote the underlying fixed size sampling design of size $m=n+k$. Note that

$$
P_{m}\left(s_{m}\right)=\sum_{s_{n}<s_{m}} P_{n}\left(s_{n}\right) Q\left(s_{m}-s_{n} \mid s_{n}\right)
$$

for all $\mathrm{s}_{\mathrm{m}}$. Let $\mathrm{S}_{\mathrm{m}}$ denote the sample space corresponding to the sampling design $P_{m}$. In section 2 we will show that for a given sampling strategy $\left(P_{n} U P_{k}, e\right)$, we can find a fixed size sampling strategy $\left(P_{m}^{*}, e^{*}\right)$ that is better.

If the sampling designs $P_{n}$ and $Q_{k}$ correspond to simple random sampling without replacement (SRSWOR) procedures, then $P_{m}$ is also an SRSWOR design. It is well known that the sampling strategy $\left(P_{m}, \bar{y}_{m}\right)$ is better than $\left(P_{n}, \bar{y}_{n}\right)$ where $\bar{y}$ is the sample mean and $P_{n}$ and $P_{m}$ are SRSWOR sampling designs of sizes $n$ and $m$ respectively. Therefore, in this situation, the use of additional resources for selecting $k$ more units is justified. This characteristic of improving the efficiency by using additional sampling units, however, is not shared by all sampling strategies. Cochran (1963), Ajgaonkar (1967), Chaudhuri (1977) and 
Chaudhuri and Mukhopadhayay (1978) considered the properties of the sample mean and/or the Horiwitz-Thompson estimator (HTE) under different sampling designs $P_{n}$ and $P_{m}$. It was observed that the sampling strategies $\left(P_{m}, H T E\right)$ and $\left(P_{m}, \bar{y}_{m}\right)$ are not necessarily better than $\left(P_{n}, H T E\right)$ and $\left(P_{n}, \bar{y}_{n}\right)$, respectively. For the case where the sampling design $Q_{k}$ is SRSWOR, Sinha (1980) presented simple conditions on the first and second order inclusion probabilities of the sampling design $P_{n}$ so that $\left(P_{n+k}\right.$, HTE) is better than $\left(P_{n+k-1}\right.$, HTE) simultaneously for a $11 k=1,2, \ldots$.

Lanke (1975) considered extending an arbitrary sampling strategy $\left(P_{n}, e_{n}\right)$ to another strategy $\left(P_{m}, e_{m}\right)$ via $Q_{k}$ so that $\left(P_{m}, e_{m}\right)$ is better than $\left(P_{n}, e_{n}\right)$ irrespective of the choice of $Q_{k}$. He proposed the estimator

$$
e_{m}\left(s_{m}\right)=\left[p_{m}\left(s_{m}\right)\right]^{-1} \sum_{s_{n} c s_{m}} e_{n}\left(s_{n}\right) p_{n}\left(s_{n}\right) Q_{k}\left(s_{m}-s_{n} \mid s_{n}\right)
$$

We will refer to the estimator (1.3) as Lanke's estimator. Notice that Lanke's estimator is in some sense Rao-Blackwellization of the estimator $e_{n}$. Lanke (1975) established that the estimator $e_{m}$ in (1.3) is at least as good as $e_{n}$ no matter what $P_{n}, e_{n}$ and $Q_{k}$ are.

Sengupta (1982) extensively studied the properties of Lanke's estimator for various choices of $e_{n}, P_{n}$ and $Q_{k}$. In particular, he observed that (i) Lanke's estimator, even though it improves over the estimator $e_{n}$, may itself turn out to be inadmissible, and ( $i i)$ if the estimator $e_{n}$ is the sample mean (or HTE) then there may not exist a sampling design $Q_{k}$ such that Lanke's estimator based on $e_{n}$ is again the sample mean (or HTE). He also showed that when $e_{n}$ is the sample mean and the sampling design $Q_{k}$ is SRSWOR, Lanke's estimator will again be the sample mean if and onty if the sampling design $P_{n}$ is itself SRSWOR. 
In Section 2, we critically review Lanke's estimator and point out some of its demerits in the present form. We then consider different versions of this estimator to explore the scope for further improvement. Section 3 contains some concluding remarks.

\section{MAIN RESULTS}

In this section, we first show that it is better to use an appropriate fixed size sampling design of total size $m=n+k$ than to use two independent sampling designs $P_{n}$ and $P_{k}$ of sizes $n$ and $k$ respectively.

Theorem 2.1 Let $P_{n} U P_{k}$ denote the sampling design obtained by taking two independent samples of sizes $n$ and $k$ using the sampling designs $P_{n}$ and $P_{k}$ respectively. Suppose $\mathrm{e}$ is an unbiased estimator for $\theta(Y)$ based on the composite sampling design $P_{n} U P_{k}$. Then, there exists a fixed size sampling design $P^{*}$ of size $m=n+k$ and an estimator $e^{*}$ such that the sampling strategy $\left(P_{m}^{*}\right.$, $\left.e^{*}\right)$ is better than $\left(P_{n} U P_{k}, e\right)$.

Proof: We assume $n+k<N$ as otherwise the claim is trivially justified. We prove the theorem by constructing the sampling strategy $\left(P_{m}^{*}, e^{*}\right)$. Define,

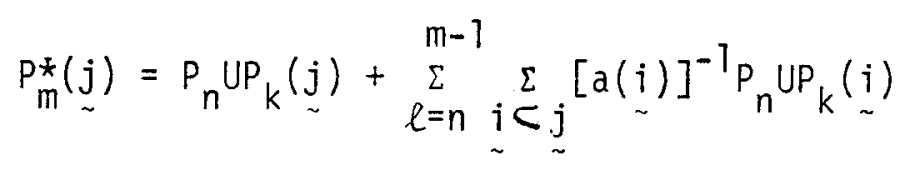

where

$$
\underset{\sim}{i}=\left(i_{1}, i_{2}, \ldots, i_{\ell}\right), \underset{\sim}{j}=\left(j_{1}, j_{2}, \ldots, j_{m}\right)
$$

and

$$
a(i)=\text { number of }{\underset{\sim}{j}}_{\sim} \text { with } P_{n} U P_{k}(\underset{\sim}{j}) \text { positive and } i \underset{\sim}{ } \underset{\sim}{j} \text {. }
$$

It is easy to see that $P_{m}^{*}$ is a fixed size sampling design of size $m=n+k$. Define, now, the estimator

$$
\left.\left.e^{*}(\underset{\sim}{j})=\left[P_{m}^{*}(\underset{\sim}{j})\right]^{-1}[e \underset{\sim}{j}) P_{n} U P_{k}(j)+\sum_{l=n}^{m-1} \underset{\sim}{i} \sum_{\sim}^{j} \underset{\sim}{j a(j)}\right\}_{\sim}^{-1} P_{n} U P_{k}(\underset{\sim}{i}) e(i)\right] .
$$


Then it is easy to verify that $e^{*}$ is unbiased for $\theta(Y)$. Also, using CauchySchwartz inequality, one can show that $\left(P_{m}^{*}, e^{*}\right)$ is better than $\left(P_{n} U P_{k}, e\right)$.

It is thus advisable to obtain additional sampling units with additional resources. Note further that given the sampling designs $P_{n}$ and $P_{k}$, the sampling design $P_{m}^{*}$ has the same form as (1.2) for some conditional sampling design $Q_{k}$.

For the remainder of this paper, we will be dealing with sampling designs of the type $P_{m}$ given by (1.2) with components given by $P_{n}$ and $\left\{Q_{k}\left(\cdot \mid s_{n}\right) s_{n} \varepsilon S_{n}\right\}$. We next show that it is not possible to obtain an estimator based on the sampling design $P_{m}$ that is better than every possible estimator based on the sampling design $P_{n}$. To give a quick demonstration of this, we restrict to homogeneous linear unbiased $(h\rceil u)$ estimators of the form $e(s, y)=\sum_{i \varepsilon S} b(i, s) Y_{i}$ and $\operatorname{set} \theta(Y)=\bar{Y}$.

Theorem 2.2: Let $P_{n}$ be a connected sampling design (see Patel and Dharmadhikari (1977)). Let $P_{m}$ be any fixed size sampling design of size $m=n+k$ obtained by extending $P_{n}$ as in (1.2) via an arbitrary $Q_{k}$. Then there does not exist any hlu estimator e based on $P_{m}$ that is (uniformly) better than every hlu estimator based on $P_{n}$.

Proof: Let $e_{1, n}, e_{2, n}, \ldots e_{N, n}$ denote a class of hlu estimators of $\bar{\gamma}$ such that the variance of the estimator $e_{i, n}$ is zero at the point $q_{i}$, where $q_{i}$ is an $\mathrm{N} \times 1$ vector with 1 in the $i$ th position and 0 elsewhere. (See Patel and Dharmadhikari (1977) to ensure the existence of such estimators).

Suppose there exists a $h$ lu estimator e based on $P_{m}$ that is better than every estimator based on $P_{n}$. Then the estimator $e$, in particular, will be better than the estimators $e_{1, n}, \ldots, e_{N, n}$. Therefore, the variance of the estimator e must be zero at the points $q_{1}, q_{2}, \ldots,{\underset{\sim}{N}}_{N}$. However, it can be easily verified that this is not possible unless e is identically $\bar{\gamma}$. 
Even though there does not exist an estimator based on $P_{m}$ that is better than every estimator based on $P_{n}$, for given sampling designs $P_{n}$ and $P_{m}$ and an estimator $e_{n}$ based on $P_{n}$, there always exists an estimator $e_{m}$ based on $P_{m}$ that is better than $e_{n}$. For example, Lanke's estimator serves this purpose. So if we wish to obtain a sampling stragegy that is better than $\left(P_{n}, e_{n}\right)$ we may use $\left(P_{m}, e_{m}\right)$ where $e_{m}$ is Lanke's estimator.

Now suppose $e_{n}$ and $f_{n}$ are two estimators for $\theta(Y)$ based on $P_{n}$. Let $e_{m}$ and $f_{m}$ denote the corresponding Lanke versions of $e_{n}$ and $f_{n}$ respectively. The following example demonstrates that even in situations where the estimator $e_{n}$ is uniformly better than the estimator $f_{n}$, it is not generally true that the estimator $e_{m}$ is better than the estimator $f_{m}$.

Example 2.1: Consider the following sampling designs and the estimators $e_{4}$ and $f_{4}$ :

\begin{tabular}{cccc}
$s_{4}$ & $P_{4}\left(s_{4}\right)$ & $f_{4}$ & $e_{4}$ \\
\hline$(1,2,3,4)$ & 0.1 & $a_{1}$ & $\left(a_{1}+a_{2}\right) / 2$ \\
$(2 ; 3,4,5)$ & 0.2 & $a_{2}$ & $\left(3 a_{2}+a_{3}\right) / 4$ \\
$(3,4,5,6)$ & 0.3 & $a_{3}$ & $\left(5 a_{3}+a_{4}\right) / 6$ \\
$(4,5,6,1)$ & 0.2 & $\dot{a}_{4}$ & $\left(3 a_{4}+a_{5}\right) / 4$ \\
$(5,6,1,2)$ & 0.1 & $a_{5}$ & $\left(a_{5}+a_{6}\right) / 2$ \\
$(6,1,2,3)$ & 0.1 & $a_{6}$ & $\left(a_{6}+a_{1}\right) / 2$ \\
\hline
\end{tabular}

where

$$
\begin{aligned}
& a_{1}=\left(280 \gamma_{1}+210 Y_{2}+140 \gamma_{3}\right) / 504 \text {, } \\
& a_{2}=\left(210 Y_{2}+140 Y_{3}+120 Y_{4}\right) / 504 \text {. } \\
& a_{3}=\left(140 Y_{3}+120 Y_{4}+140 Y_{5}\right) / 504 \text {, } \\
& a_{4}=\left(120 \gamma_{4}+140 \gamma_{5}+210 \gamma_{6}\right) / 504 \\
& a_{5}=\left(140 \gamma_{5}+210 \gamma_{6}+280 \gamma_{1}\right) / 504 \\
& a_{6}=\left(210 Y_{6}+280 Y_{1}+210 Y_{2}\right) / 504
\end{aligned}
$$


It is easy to show that the estimate $e_{.4}$ is better than the estimator $f_{4}$. Consider now the following extension of the sampling design and the corresponding Lanke's estimators:

\begin{tabular}{|c|c|c|c|c|c|c|}
\hline$s_{4}$ & $\begin{array}{l}\text { Extension } \\
\text { with } k=1\end{array}$ & $Q_{1}(\cdot 1 \cdot)$ & $s_{5}$ & $P_{5}\left(s_{5}\right)$ & $f_{5}$ & $e_{5}$ \\
\hline \multirow[t]{2}{*}{$(1,2,3,4)$} & 5 & 0.5 & \multirow[t]{2}{*}{$(1,2,3,4,5)$} & \multirow[t]{2}{*}{0.15} & $\left(a_{1}+2 a_{2}\right)$ & $\left(a_{1}+4 a_{2}+a_{3}\right)$ \\
\hline & 6 & 0.5 & & & 3 & 6 \\
\hline \multirow[t]{2}{*}{$(2,3,4,5)$} & 6 & 0.5 & \multirow[t]{2}{*}{$(2,3,4,5,6)$} & \multirow[t]{2}{*}{0.25} & $\left(2 a_{2}+3 a_{3}\right)$ & $\left(3 a_{2}+6 a_{3}+a_{4}\right)$ \\
\hline & 1 & 0.5 & & & 5 & 10 \\
\hline \multirow[t]{2}{*}{$(3,4,5,6)$} & 1 & 0.5 & \multirow[t]{2}{*}{$(3,4,5,6,7)$} & \multirow[t]{2}{*}{0.25} & $\left(3 a_{3}+2 a_{4}\right)$ & $\left(5 a_{3}+4 a_{4}+a_{5}\right)$ \\
\hline & 2 & 0.5 & & & 5 & 10 \\
\hline \multirow[t]{2}{*}{$(4,5,6,1)$} & 2 & 0.5 & \multirow[t]{2}{*}{$(4,5,6,1,2)$} & \multirow[t]{2}{*}{0.15} & $\left(2 a_{4}+a_{5}\right)$ & $\left(3 a_{4}+2 a_{5}+a_{6}\right)$ \\
\hline & 3 & 0.5 & & & 5 & 6 \\
\hline \multirow[t]{2}{*}{$(5,6,1,2)$} & 3 & 0.5 & \multirow[t]{2}{*}{$(5,6,1,2,3)$} & \multirow[t]{2}{*}{0.10} & $\left(a_{5}+a_{6}\right)$ & $\left(a_{5}+2 a_{6}+a_{1}\right)$ \\
\hline & 4 & 0.5 & & & 2 & 4 \\
\hline \multirow[t]{2}{*}{$(6,1,2,3)$} & 4 & 0.5 & \multirow[t]{2}{*}{$(6,1,2,3,4)$} & \multirow[t]{2}{*}{0.10} & $\left(a_{6}+a_{7}\right)$ & $\left(a_{6}+2 a_{1}+a_{2}\right)$ \\
\hline & 5 & 0.5 & & & 2 & 4 \\
\hline
\end{tabular}

It can be shown that the variance of $f_{5}$ is smaller than that of $e_{5}$ at the point $q=(0,0,0,0,1,0)$ and hence $e_{5}$ is not better than $f_{5}$.

In the next example we demonstrate that Lanke's extension of an admissible estimator may turn out to be inadmissible. This was first demonstrated by Sengupta (1982). We, however, present a different example here. 
Example 2.2: Consider the following sampling designs for a population of size six.

\begin{tabular}{cccccc}
$s_{3}$ & $P_{3}\left(s_{3}\right)$ & $\begin{array}{c}\text { Extension } \\
\text { with } k=2\end{array}$ & $Q_{2}$ & $s_{5}$ & $P_{5}\left(s_{5}\right)$ \\
\hline$(1,2,3)$ & 0.1 & $(4,5)$ & 1 & $(1,2,3,4,5)$ & 0.31 \\
$(1,2,5)$ & 0.2 & $(3,6)$ & 1 & $(1,2,3,5,6)$ & 0.20 \\
$(2,4,6)$ & 0.1 & $(3,5)$ & 1 & $(2,3,4,5,6)$ & 0.10 \\
$(2,3,4)$ & 0.3 & $(1,6)$ & 1 & $(1,2,3,4,6)$ & 0.30 \\
$(1,3,5)$ & 0.3 & $(4,6)$ & 0.3 & $(1,3,4,5,6)$ & 0.09 \\
\hline
\end{tabular}

Let $e_{3}$ denote the HTE for $\theta(Y)$ based on $P_{3}$. Consider the estimator

$$
\begin{aligned}
& e(1,2,3,4,5)=\mathrm{ae}_{3}(1,2,3)+b \mathrm{e}_{3}(1,3,5)+c e_{3}(2,3,4) \\
& e(1,2,3,4,6)=d e_{3}(2,3,4)+\mathrm{ee} \mathrm{e}_{3}(1,2,3)+f \mathrm{e}_{3}(2,4,6) \\
& \mathrm{e}(2,3,4,5,6)=g \mathrm{e}_{3}(2,4,6)+h \mathrm{e}_{3}(2,3,4) \\
& \mathrm{e}(1,3,4,5,6)=\mathrm{e}_{3}(1,3,5) \\
& \mathrm{e}(1,2,3,5,6)=\mathrm{ie} \mathrm{e}_{3}(1,2,5)+j \mathrm{e}_{3}(1,2,3)+\mathrm{ke}_{3}(1,3,5) .
\end{aligned}
$$

Note that Lanke's extension of $e_{3}$ is obtained by setting $a=\frac{10}{31}, b=\frac{21}{31}, d=g=i=1$ and $c=e=f=h=j=k=0$.

However, it is easy to show that the estimator e with $a=\frac{10}{31}, b=\frac{21}{31}, c=0, d=\frac{3}{4}$, $\mathrm{e}=0, f=\frac{1}{4}, g=\frac{1}{4}, h=\frac{3}{4}, i=1$ and $j=k=0$ is better than Lanke's

estimator. Infact one can construct several other estimaotrs that are better than Lanke's estimator.

Note that in the above example (as in any other example) Lanke's estimator assigns non-zero weight to only those subsamples $s_{n}$ of $s_{m}$ for which $Q_{k}\left(s_{m}-s_{n} \mid s_{n}\right)$ is positive. However, positive weights could be assigned to all subsamples $s_{n}$ of $s_{m}$ for which $P_{n}\left(s_{n}\right)$ is positive. We investigate this possibility below and present some improvements over Lanke's estimator. Consider 


$$
e_{m}\left(s_{m}\right)=\sum_{n} \sum_{m} e_{n}\left(s_{n}\right) W\left(s_{n}, s_{m}\right)
$$

where $\left\{W\left(s_{n}, s_{m}\right)\right\}$ are nonnegative constants. Note that if

$$
\sum_{m} s_{n} W\left(s_{n}, s_{m}\right) P_{m}\left(s_{m}\right)=P_{n}\left(s_{n}\right)
$$

for all $s_{n}$, then the estimator $e_{m}$ is unbiased for $\theta(Y)$ whenever $e_{n}$ is so. Also, using Cauchy-Schwartz inequality, it can be shown that the estimator $e_{m}$ is as good as $e_{n}$ if

$$
\sum_{s_{m}>s_{n}} W\left(s_{n}, s_{m}\right) P_{m}\left(s_{m}\right)\left\{s_{n}^{i} s_{m} s_{m} W\left(s_{n}^{\prime}, s_{m}\right\} \leq p_{n}\left(s_{n}\right)\right.
$$

for all $s_{n}$. It is now easy to see that, under the condition (2.3), (2.4) holds, if and only if,

$$
s_{n} \in s_{m} W\left(s_{n}, s_{m}\right)=1
$$

for al1 $\mathrm{s}_{\mathrm{m}}$. We can also express the estimator $e_{m}$ in (2.2) as

$$
e_{m}\left(s_{m}\right)=\left[P_{m}\left(s_{m}\right)\right]^{-1} \sum_{n} c s_{m} e_{n}\left(s_{n}\right) P_{n}\left(s_{n}\right) Q_{k}^{*}\left(s_{m}-s_{n} \mid s_{n}\right)
$$

where $Q_{k}^{*}$ is given by

$$
Q_{k}^{*}\left(s_{m}-s_{n} \mid s_{n}\right)=P_{m}\left(s_{m}\right)\left[P_{n}\left(s_{n}\right)\right]^{-1} W\left(s_{m}, s_{n}\right)
$$

Then, from (2.3) and (2.5) we get

$$
s_{m} \sum_{s_{n}} Q_{k}^{*}\left(s_{m}-s_{n} \mid s_{n}\right)=1 \text {, for all } s_{n}
$$

and

$$
\sum_{n} c s_{m} P_{n}\left(s_{n}\right) Q_{k}^{*}\left(s_{m}-s_{n} \mid s_{n}\right)=P_{m}\left(s_{m}\right)
$$

for all $\mathrm{s}_{\mathrm{m}}$.

In summary, the general extension $e_{m}$ in (2.2) that improves over the estimator $e_{n}$ has the same form as Lanke's estimator. The only difference is that the estimator(2.2) uses possibly a different sampling design $Q_{k}^{*}$ generating the 
same final design $P_{m}$. In some situations it is possible to find a different $Q_{k}^{*}$ so that the estimator $(2.2)$ is better than Lanke's estimator computed with $Q_{k}$. The choice of $Q_{k}^{*}$ would be such that the estimator (2.2) assigns positive weights to al1 $e_{n}\left(s_{n}\right)$ for which $s_{n} s_{m}$ and $P_{n}\left(s_{n}\right)>0$. We now present one such choice in the next theorem.

Theorem 2.3: Suppose the sampling designs $P_{n}, Q_{k}$ and $P_{m}$ are as defined in section 1 . Define,

$$
A\left(s_{m}\right)=\left\{s_{n}: s_{n} c_{m}, Q_{k}\left(s_{m}-s_{n} \mid s_{n}\right)>0\right\}
$$

and

$$
B\left(s_{m}\right)=\left\{s_{n}: s_{n} \in s_{m}, Q_{k}\left(s_{m}-s_{n} j s_{n}\right)=0\right\} .
$$

Suppose there exist two samples $s_{m}(1)$ and $s_{m}(2)$ such that

(i) $A\left(s_{m}^{(1)}\right) \cup B\left(s_{m}^{(1)}\right)=A\left(s_{m}^{(2)}\right) \cup B\left(s_{m}^{(2)}\right)$.

and

(ii) both $A\left(s_{m}{ }^{(1)}\right) \cap B\left(s_{m}{ }^{(2)}\right)$ and $A\left(s_{m}{ }^{(2)}\right) \cap B\left(s_{m}{ }^{(1)}\right)$ are not empty.

Then Lanke's estimator $e_{m}\left(s_{m}\right)$ in (1.3) is inadmissible.

Proof: $\quad$ Define

$$
e_{m}^{*}\left(s_{m}\right)=\left\{\begin{array}{l}
e_{m}\left(s_{m}\right) \quad \text { for } s_{m} \neq s_{m}(1) \text { or } s_{m}(2) \\
{\left[P_{1} e_{m}\left(s_{m}(1)\right)+P_{2} e_{m}\left(s_{m}^{(2)}\right)\right] /\left(P_{1}+P_{2}\right) \text { if } s_{m}=s_{m}(1) \text { or } s_{m}}
\end{array}\right.
$$

when $e_{m}\left(s_{m}\right)$ is Lanke's estimator in (1.3) and $P_{i}=P_{m}\left(s_{m}(i)\right) i=1,2$. It is easy to verify that $e_{m}^{*}$ is better than $e_{m}$ and hence $e_{m}$ is inadmissible.

Note that Lanke's estimator $e_{m}$ puts zero weight to $e_{n}\left(s_{n}\right)$ for $s_{n}$ in $B\left(s_{m}(1)\right.$ ) and $B\left(s_{m}{ }^{(2)}\right)$ whereas the estimator in (2.9) assigns positive weights. Note also that the estimator in (2.9) is Lanke-type estimator with a different choice of $Q_{k}^{*}$. In fact, 


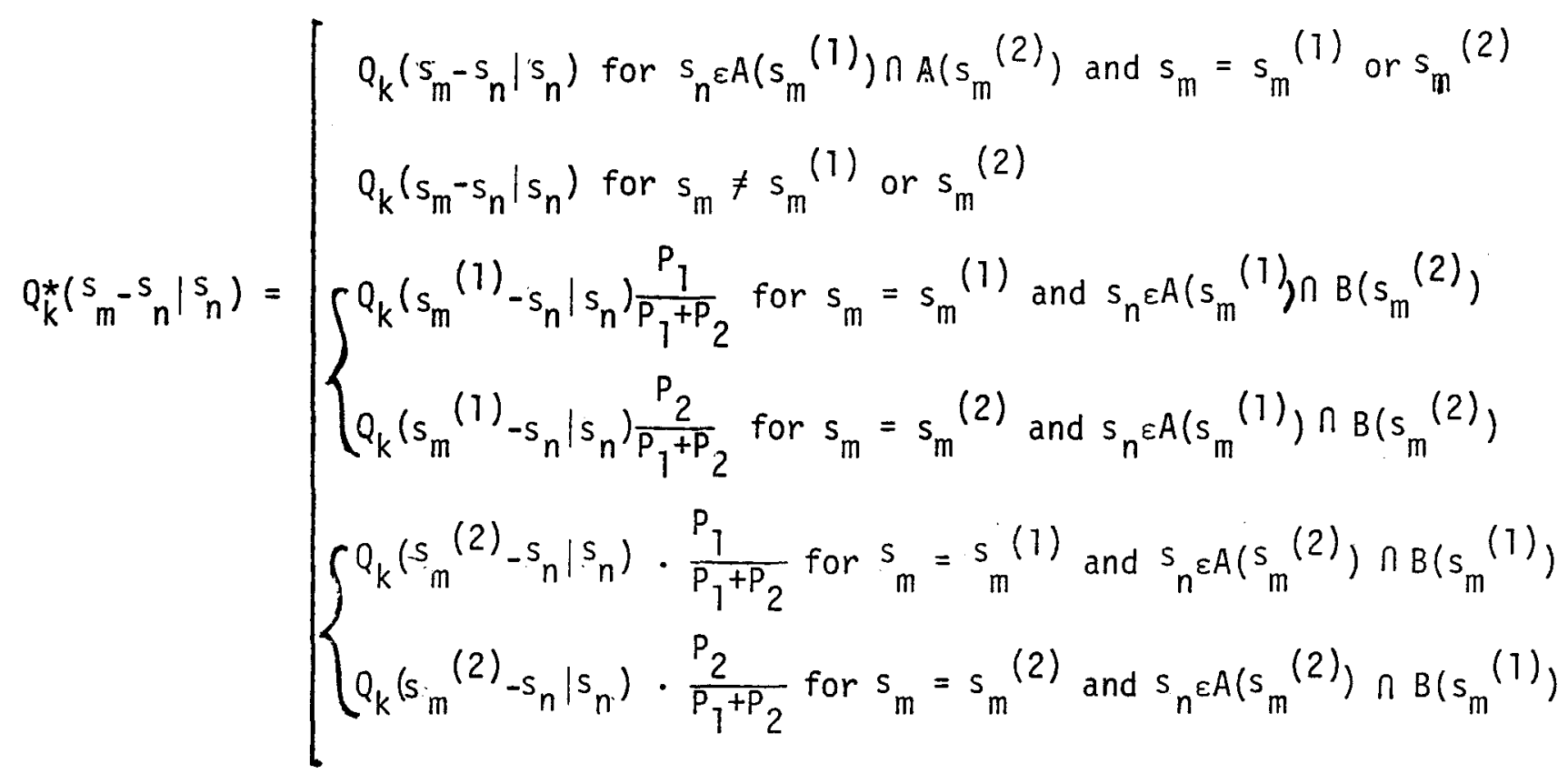

The conditions for applicability of the above theorem mean that every $s_{n} \varepsilon A\left(s_{m}(1)\right.$ ) $U A\left(s_{m}^{(2)}\right)$ is a subset of both $s_{m}(1)$ and $s_{m}{ }^{(2)}$. Lanke's estimator seems to distinguish between the two samples $s_{m}(1)$ and $s_{m}(2)$ while using $e_{n}\left(s_{n}\right)$. On the other hand, the revised estimator $e_{m}^{*}\left(s_{m}\right)$ does, in fact, the 'averaging' or 'unordering' and, consequently, performs better than that of Lanke. The generalization of this result to other complicated 'structures' is not difficult and hence is not included here. However, the improved estimator is seen to be again a Lanke type estimator with a revised extension rule $Q_{k}^{*}$ but with the same over-all sampling design $P_{m}$. 


\section{CONCLUDING REMARKS}

The following observations have been made in this paper.

(a) Lanke's formula yields a strategy $\left(P_{m}, e_{m}\right)$ which is better than any given strategy $\left(P_{n}, e_{n}\right)$ irrespective of the choice of the extension rule $Q_{k}$ where $m=n+k$. Also, for any given strategy $\left(P_{n} U P_{k}, e\right)$ governed by a combination of two independent sampling designs $P_{n}$ and $P_{k}$, there exists a strategy $\left(P_{m}^{*}, e^{*}\right)$ which performs better.

(b) It is difficult to set out the estimator at the initial stage as the ordering is not generally preserved by Lanke type improved estimators.

(c) Lanke's formula may sometimes lead to inadmissible estimators due to faulty selection of the extension rule $Q_{k}$. The structure of the samples underlying $P_{n}$ and $P_{m}$ may be studied and suitable recommendations made in some cases.

The following problems need further investigation:

(i) Order-preserving improved estimators using suitable/given extension rules.

(ii) Admissible improved estimators using suitable given extension rules. As mentioned earlier, Sinha (1980) and Sengupta (1982) have some interesting preliminary results on characterizations of original sampling strategies ensuring (ii) with the extension rules given by SRSWOR designs. Is it possible to construct improved estimators in general terms which are essentially different from those given by Lanke's formula? 


\section{REFERENCES}

Chaudhuri, A. (1977). On the Choice of Sample Size for a HorvitzThompson Estimator, Jour. Indian Soc. Agri. Stat., 29, $35-42$.

Chaudhuri, A. and Mukhopadhyay, P. (1978): A note on how to choose the sample size for Horvitz-Thompson estimation. Calcutta Statisti. Assoc. Bu11., 27, 149-154.

Cochran, W. G. (1963): Sampling Techniques, 2nd ed. John Wiley, New York.

Lanke, J. (1975): Some contributions to theory of survey sampling Ph.D. Thesis, University of Lund, Sweden.

Patel, H. C. and Dharmadhikari, S. W. (1977). On linear invariant unbiased estimators in survey sampling. Sankhyä Series C, $39,21-27$.

Prabhu-Ajgaonkar, S. G. (1967): The effect of increasing sample size on the precision of an estimator. Amer. Statistician, $21(4), 26-28$.

Sengupta, S. (1982): Further studies on some strategies in sampling finite populations. Unpublished Ph.D. Thesis, Calcutta University.

Sinha, B. K. (1980): On the concept of admissible extensions of sampling designs and some related problems. Tech. Report, No. 14/80, Stat-Math. Division, Indian Statistical Institute, Calcutta. 